\title{
Reflets
}

Revue d'intervention sociale et communautaire

\section{Regards communautaires : le projet du Boisé comme vecteur de citoyenneté}

Katharine Larose-Hébert, Gabrielle Alexandre, Caroline Bastien, Amanda Blouin, Karina Boucher, Myriam Boudreault, Eren Buyukbicer, Jean Charette, Janik Clément, Stéphanie Côté, Vanessa Couturier, Mathieu Déziel-Parent, John Flynn, Michèle Frenette, Charlotte Gagnon, Andrea Gray Kegevic, Rachael John-West, Lauren Kluke, Jessica Kozlowski, Alexandra Laroche, Philippe Le Voguer, Vanessa Major, Aude Martel, Jessica Morris, Clarence Kapinga Nkuba, Émilie Marie Santostefano, Véronique Savard, Emily Tait et Rachelle Thibert

Volume 22, numéro 1, printemps 2016

URI : https://id.erudit.org/iderudit/1037168ar

DOI : https://doi.org/10.7202/1037168ar

Aller au sommaire du numéro

Éditeur(s)

Reflets, Revue d'intervention sociale et communautaire

ISSN

1203-4576 (imprimé)

1712-8498 (numérique)

Découvrir la revue

Citer cet article

Larose-Hébert, K., Alexandre, G., Bastien, C., Blouin, A., Boucher, K., Boudreault, M., Buyukbicer, E., Charette, J., Clément, J., Côté, S., Couturier, V., Déziel-Parent, M., Flynn, J., Frenette, M., Gagnon, C., Kegevic, A. G., John-West, R., Kluke, L., Kozlowski, J., Laroche, A., Le Voguer, P., Major, V., Martel, A., Morris, J., Nkuba, C. K., Santostefano, É. M., Savard, V., Tait, E. \& Thibert, R. (2016). Regards communautaires : le projet du Boisé comme vecteur de citoyenneté. Reflets, 22(1), 183-193. https://doi.org/10.7202/1037168ar

Tous droits réservés (c Reflets, Revue d'intervention sociale et communautaire, 2016
Ce document est protégé par la loi sur le droit d'auteur. L’utilisation des services d’Érudit (y compris la reproduction) est assujettie à sa politique d'utilisation que vous pouvez consulter en ligne. 


\section{Regards communautaires : le projet du Boisé comme vecteur de citoyenneté}

Katharine Larose-Hébert, Ph.D.

Stagiaire postdoctorale, professeure remplaçante

École de service social, Université d'Ottawa

avec la collaboration des coauteures et coauteurs suivants :

Gabrielle Alexandre, Caroline Bastien, Amanda Blouin, Karina

Boucher, Myriam Boudreault, Eren Buyukbicer, Jean Charette, Janik

Clément, Stéphanie Côté, Vanessa Couturier, Mathieu Déziel-

Parent, John Flynn, Michèle Frenette, Charlotte Gagnon, Andrea

Gray Kegevic, Rachael John-West, Lauren Kluke, Jessica Kozlowski, Alexandra Laroche, Philippe Le Voguer, Vanessa Major, Aude Martel, Jessica Morris, Clarence Kapinga Nkuba, Émilie Marie Santostefano, Véronique Savard, Emily Tait, Rachelle Thibert

\section{Introduction}

Le présent article est issu d'une recherche dont l'objectif était de contribuer à l'évaluation du projet pilote du Boisé en s'appuyant sur l'expérience des acteurs et actrices du milieu communautaire. Ce projet de campement urbain saisonnier a nécessité la modification des règlements municipaux quant à l'occupation d'un espace public sur les berges du ruisseau de la Brasserie, dans le quartier du Vieux-Hull, par des citoyens et citoyennes, majoritairement des personnes en situation d'itinérance qui souhaitaient y résider pour la saison estivale. La collecte de données a été effectuée auprès de 11 personnes, toutes employées par l'un des six organismes communautaires partenaires de la recherche 
afin de partager leur expérience et leur évaluation du projet. Dans l'ensemble, les participants à la recherche estiment que le projet a contribué à améliorer les conditions de vie des personnes installées dans le campement (les " campeurs ") et à leur donner une voix, mais ils portent un regard critique sur ses limites, surtout en ce qui a trait au profilage social des populations marginalisées. Ils reconnaissent que le Boisé ne représente pas une solution à long terme pour la lutte contre la pauvreté et l'itinérance. Malgré ce fait, la majorité d'entre eux considèrent que la poursuite du projet est souhaitable.

Cette recherche est le fruit du travail collaboratif de 28 étudiants et étudiantes de quatrième année du baccalauréat spécialisé approfondi en service social offert à l'Université d'Ottawa. Dans le cadre de leur cours de Recherche communautaire et recherche-action en service social, suivi à la session d'hiver 2015, l'ensemble du groupe-classe a mené une recherche visant à contribuer à l'évaluation de ce projet pilote développé à Gatineau et communément appelé le projet du "Boisé ». Ce projet de recherche a été élaboré conjointement par leur professeure et l'organisme partenaire principal, Droits-Accès de l'Outaouais (DAO), avec la contribution de cinq organismes communautaires impliqués de manière directe ou indirecte dans la mise en œuvre du Boisé, soit l'Association pour la défense des droits sociaux (ADDS), le Bureau régional d'action sida (BRAS), le Centre d'intervention et de prévention en toxicomanie de l'Outaouais (CIPTO), le Collectif régional de lutte à l'itinérance en Outaouais (CRIO) et la Soupe populaire de Hull.

Le projet du Boisé a été officiellement mis en œuvre au cours de l'été 2014, mais ce lieu quelque peu isolé, situé à proximité des berges du ruisseau de la Brasserie, était occupé par des personnes en situation de marginalité depuis de nombreuses années déjà. Le développement de ce "camping urbain " aura nécessité des interventions soutenues de la part des partenaires communautaires ci-nommés et des usagers de leurs services. En effet, ils ont dû convaincre les instances institutionnelles (municipalité, service de police et Agence de la santé et des services sociaux), initialement réticentes au projet, d'investir dans celui-ci et d'y apporter leur 
appui. Cette initiative, d'abord et avant tout citoyenne, visait à démocratiser l'occupation des espaces publics, ainsi qu'à revendiquer le droit à un hébergement sécuritaire pour tous. Son émergence est fortement liée au contexte actuel de pénurie de logements abordables ou de transition, au manque ou à l'absence de services adaptés aux personnes en situation d'itinérance et, globalement, à la précarisation sociale des populations marginalisées pouvant être constatée dans la région de l'Outaouais, comme dans celle d'Ottawa ainsi qu'ailleurs au Canada. Le milieu communautaire s'est investi dans le démarrage de ce projet afin de soutenir les doléances de leurs usagers désireux de résider dans cet espace, en les accompagnant dans une démarche d'empowerment et de défense de droits.

Dans le présent article, nous passerons d'abord en revue très brièvement la littérature sur le sujet de l'itinérance. Nous présenterons ensuite les questions ayant guidé cette recherche, ainsi que le modèle évaluatif auquel nous nous sommes référés pour nos analyses. Enfin, nous exposerons la méthodologie utilisée, suivie des résultats et de leur analyse.

\section{Problématisation}

Le phénomène de l'itinérance n'est pas simple à définir, ni à décrire. Il est lié à divers enjeux structurels, personnels et sociaux, dont les dynamiques sont en interaction et donc indissociables. Ce processus longitudinal de désaffiliation sociale est marqué par un cumul de ruptures dans les espaces économique, relationnel et symbolique. Le terme "itinérance » ne possède pas nécessairement une connotation négative en soi; il fait référence au déplacement, au mouvement et à l'absence de sédentarité. Toutefois, dans le contexte actuel, il est fortement associé à l'urbanité, à la pauvreté et à la marginalité; il s'oppose à la normativité sociale. Ainsi, une personne en situation d'itinérance aura une situation de vie caractérisée par l'extrême pauvreté et l'instabilité en matière de logement et adoptera souvent des comportements hors normes. 
Selon Campeau (2000), il existe deux principaux modèles explicatifs de l'itinérance : l'un qui repose sur des facteurs individuels et l'autre qui cible des facteurs structurels. Toutefois, l'auteur souligne que l'explication individuelle déresponsabilise la collectivité face à ce phénomène. L'itinérance découle plus directement de la manière dont les ressources de la société sont organisées et distribuées. L'enjeu du logement est prédominant dans cette problématique. En effet, une disparité importante peut être constatée entre les revenus des personnes en situation d'itinérance et le prix des loyers. La pénurie de logements abordables fait en sorte que le prix moyen des logements représente souvent plus $50 \%$ des revenus des prestataires des programmes de dernier recours, ce qui a pour effet de vulnérabiliser ce groupe de la population. Laberge et Roy (2001) rappellent qu'une participation citoyenne à part entière débute par un domicile fixe.

\section{Judiciarisation de l'itinérance}

La construction sociale des espaces publics influence directement les regards que porte la société sur les groupes marginaux qui les occupent. Devant l'absence d'un espace privé, les personnes en situation d'itinérance sont contraintes à présenter leur "visibilité " dérangeante. Devant la transgression des normes sociales relatives à l'utilisation de l'espace public (uriner, faire le trafic de drogues, être en état d'ébriété, dormir, etc.), les policiers utilisent des mesures coercitives. Ainsi, le processus de judiciarisation des personnes marginalisées débute souvent par des interventions policières dans les espaces publics. Une des stratégies utilisées par les policiers afin de "mettre à l'écart " les personnes déviant de l'ordre normatif comportemental est d'affirmer que leur présence nuit à l'ordre public. Des contraventions et des constats d'infraction sont donc émis à répétition pour des violations mineures aux règlements municipaux ou au Code criminel. Cette criminalisation des stratégies de survie des populations vulnérables met en lumière les relations de pouvoir sous-jacentes à l'utilisation du droit comme modalité de régulation de la déviance. Les personnes en situation d'itinérance font face à divers obstacles pour faire valoir leurs droits. Leurs parcours d'infortune, découlant bien souvent des 
structures mêmes de la société, et leurs expériences d'exclusion sont le reflet des inégalités et des injustices perpétuées par les classes privilégiées. Leur lutte pour la reconnaissance de leur citoyenneté doit donc être soutenue par l'ensemble des acteurs et actrices du milieu social et ultimement par la société entière.

\section{Modèle d'évaluation et questions de recherche}

Notre recherche évaluative s'est inspirée très librement de Brousselle, Champagne, Contandriopoulos et Hartz (2011), et plus spécifiquement de leur modèle d'analyse logique. Nous définirons brièvement les concepts auxquels nous nous sommes référés pour procéder à cette évaluation. Selon les auteurs susmentionnés :

« évaluer» consiste fondamentalement à porter un jugement de valeur sur une intervention en mettant en œuvre un dispositif capable de fournir des informations scientifiquement valides et socialement légitimes sur cette intervention ou sur n'importe laquelle de ses composantes (p.52).

Ils indiquent que l'objectif sous-jacent à l'évaluation est " de faire en sorte que les différents acteurs concernés, dont les champs de jugement sont parfois différents, soient en mesure de prendre position sur l'intervention pour qu'ils puissent construire, individuellement ou collectivement, un jugement susceptible de se traduire en actions. " (Brousselle, et collab, 2011, p. 52).

Selon Contandriopoulos et collab. (2000), la recherche évaluative est une démarche qui permet de poser un jugement « après les faits » sur une intervention en employant des méthodes scientifiques. Plus spécifiquement, il s'agit d'analyser la pertinence, les fondements théoriques, la productivité, les effets et le rendement d'une intervention ainsi que les relations existantes entre l'intervention et le contexte dans lequel elle se situe, généralement dans le but de mieux comprendre l'intervention et d'aider à la prise de décision. Elle produit des effets cohérents avec la logique théorique et, d'autre part, vise à améliorer les 
stratégies d'intervention pour qu'elles produisent des effets optimaux (p. 11). Ainsi, afin d'évaluer une intervention, il faut comparer la conception du projet par rapport à la réalisation du projet. Est-ce que ce qui était prévu est en fait arrivé? Est-ce que les résultats de cette intervention ont créé les bénéfices escomptés? Pour répondre à ces questions, nous avons interrogé 11 employés des six organismes communautaires ayant accepté de participer à notre recherche. Dans la section suivante, nous présenterons les étapes de notre méthodologie.

\section{Méthodologie}

Les 28 étudiants et étudiantes du cours se sont réparti la collecte de données par équipe. Ainsi, chaque équipe, constituée de deux ou trois personnes, a rencontré dans le cadre d'un entretien semidirigé un des participants à la recherche. Chaque participant était majeur et employé $(n=10)$ ou ayant été employé $(n=1)$ d'un des six organismes au cours de l'été 2014, soit lors du déroulement de l'intervention. Notre échantillon est composé de huit hommes et trois femmes. Les postes occupés par les participants variaient d'intervenant à directeur général. Au moment de l'intervention, six des participants occupaient des postes de gestion et cinq, des postes d'intervention.

Les dix catégories thématiques ayant servi au codage des données étaient les suivantes : 1) géographie/territoire; 2) problèmes sociaux derrière l'intervention du Boisé; 3) raisons invoquées pour mettre en place ce projet; 4) disposition spatiale du campement; 5) actions des acteurs et actrices issus du milieu communautaire; 6) appréciation de l'intervention policière; 7) relations entre les divers partenaires; 8 ) description des campeurs; 9) évaluations personnelles des participants;10) recommandations des participants. Nous vous proposerons ci-après un très bref aperçu des résultats et de l'analyse de ceux-ci. 


\section{Résultats}

De façon unanime, les participants interviewés s'entendent pour dire que la pauvreté est la raison principale pour laquelle le campement du Boisé, en tant qu'option de logement, a été " créé ». Effectivement, le quartier du Vieux-Hull compte davantage de personnes et de familles vivant sous le seuil de la pauvreté qu'ailleurs à Gatineau, voire en Outaouais (Ville de Gatineau, 2006).

"La Ville et les autres acteurs repoussent les causes de la pauvreté sur les individus au lieu $[\ldots]$ pis j'le comprends parce que comme tout bon capitaliste, ils veulent pas dénoncer ce qu'eux-mêmes créent, mais pour nous, y est là le problème. » (Participant 9)

Selon les participants, le projet offre assurément des avantages aux campeurs et contribue à leur bien-être de façons multiples : une plus grande sécurité, une certaine hygiène, des moments de loisir, la création d'un réseau social, l'établissement de liens significatifs avec les intervenants communautaires permettant, par exemple, le développement de liens de confiance et la réduction des méfaits.

"Le Boisé a été naturellement choisi par les gens parce qu'il est à proximité des organismes et services du centre-ville, et parce qu'ils se font moins achaler par la police que dans des endroits publics comme des parcs. Aussi, s'il y a des arrestations, ils ont des témoins, du fait qu'ils sont regroupés tous ensemble.» (Participant 10)

«Le Boisé offre des avantages pour ces gens [...] ils ont accès à des services qui sont de base [...] Ils laissent aller le logement l'été. Le logement, $\mathrm{j}$ 'dis un logement, des fois c'est une chambre dans un logement à huit, avec un, deux par 
chambre. Des fois, c'est des chambreurs dans des maisons de chambres qui disent : 'Ben écoutez pendant trois, quatre, cinq mois j'va sauver un peu d'argent sur ma chambre pis j'va pouvoir mieux m'nourrir. J'va pouvoir peut-être un p'tit peu m'divertir.' Parce qu'ils ont le droit de se divertir aussi. » (Participant 8)

Selon une recherche menée sur l'itinérance dans la région de l'Outaouais, les personnes en situation d'itinérance n'ont pas d'espace leur permettant tout simplement d'être (Cyr, 2011). Le Boisé, bien qu'aujourd'hui encadré d'une certaine manière par une entente officielle, offre un lieu qui ne soit pas entièrement régi par les dictats des normes sociales dominantes, qu'elles soient issues de l'intervention, de la santé ou de la sécurité, par exemple : un espace public et personnel à la fois.

«C'est une espèce d'autorégulation qui s'est faite dans le Boisé par les campeurs pis c'est vraiment intéressant à voir parce que, pour une fois, on parlait d'un espace de liberté. » (Participant 7)

«Un projet par et pour, donc ces gens-là, c'est eux-mêmes qui s'organisent, puis nous autres on veille à ce que ce qu'on a mis en place soit effectif, puis que ça prenne forme. » (Participant 6)

Cependant, bien que plusieurs bénéfices aient pu être constatés, l'un des enjeux soulevés a été le risque de ghettoïsation qui encouragerait le profilage social.

«[...] ce projet-là, on a peur que ça finisse par faire un ghetto, que ça favorise le profilage social parce que la police les a tous sous les yeux à même place; nous, notre crainte, c'est ça là. » (Participant 4)

Étant donné que tous les citoyens et citoyennes ont droit à un logement décent, répondant à leurs besoins, et qu'une tente n'est 
pas en tant que tel un logement idéal, les participants ont tous tenu à mentionner qu'ils poursuivront leurs revendications militantes.

"En Outaouais, bon là regarde. Il faut des logements sociaux, des logements qui soient conçus, créés, que les critères d'admissibilité soient pensés et conçus avec les personnes. (Participant 8)

"On a une crise de logement social; les logements sont pas adéquats, puis on n'a pas de place pour héberger tous ces gens-là; ça fait que ce qui arrive, c'est comment que le Boisé est venu au monde.» (Participant 6)

\section{Analyse}

Le projet du Boisé met en lumière les "manques de services " observés dans le réseau de services de santé et de services sociaux, ainsi que la multitude de besoins auxquels les services actuels ne peuvent pas répondre ou ne parviennent pas à satisfaire. Bien que le projet du Boisé ne soit pas sans lacune, son existence même nous offre la possibilité d'accroître notre compréhension des dynamiques sous-jacentes à sa création et contribue à stimuler la lutte contre la pauvreté et l'itinérance dans la région de l'Outaouais.

Selon la majorité des participants à cette recherche, le projet du Boisé a eu des retombées plutôt positives pour les campeurs, ce qui s'aligne sur leur théorie initiale. En effet, il semblerait que le projet ait permis aux campeurs de prendre la parole et de reprendre un certain contrôle sur leur vie. Dans le même sens, le projet a permis aux campeurs de s'organiser et de s'impliquer dans un projet qui leur appartenait. Cela démontre le contrôle qu'ils ont pris sur ce projet, puisqu'ils se le sont approprié. Comme l'explique Le Bossé (2003), en ce qui concerne l'empowerment, il est important que les personnes touchées par une problématique soient placées au cœur de la définition du changement anticipé, pour qu'elles 
puissent exercer un pouvoir d'influence sur la situation. Afin de favoriser l'empowerment, ces personnes doivent alors être impliquées concrètement dans l'initiative sociale en question, comme cela a été le cas dans le projet du Boisé.

Grâce à l'autorégulation et à l'accompagnement, le Boisé peut être un lieu où ces personnes pourraient renouer avec la communauté et reprendre le pouvoir sur leur situation. Selon certains participants, plusieurs campeurs ont vécu une expérience positive à cet effet. Ainsi, malgré certains aspects contradictoires de l'intervention dans son ensemble (par exemple l'aspect de surveillance et de contrôle par les autorités), le projet aura contribué à l'épanouissement et au développement d'un sentiment d'autoréalisation chez certains campeurs.

Quant aux effets moins enviables du projet, il va sans dire que les intervenants et intervenantes n'avaient pas l'intention de créer une telle ghettoïsation en regroupant des personnes en situation d'itinérance ou de marginalité dans un même endroit. Quoique ce regroupement ait facilité l'accès des intervenants et intervenantes à la population vulnérable du quartier, le profilage social s'est effectué plus facilement par les policiers, tout comme le contrôle des campeurs par la municipalité. L'accès facile des instances publiques au Boisé a, en quelque sorte, brimé la vie privée des campeurs. Les personnes en situation d'itinérance sont sur un genre de territoire "emprunté " lorsqu'elles se situent dans un endroit public (Cyr, 2011). Cependant, malgré ces écueils, il est indéniable que le projet du Boisé, dans la forme que le milieu communautaire privilégie et sans être une réponse à la problématique de l'itinérance, offre du moins un espace à des personnes souvent oubliées par les politiques publiques et contribue de ce fait à l'appropriation du pouvoir des citoyens et citoyennes qui y résident et y résideront.

Finalement, plusieurs participants ont noté le maintien du profilage social dans ce quartier défavorisé et ont souligné le risque d'accroissement de cette pratique discriminatoire par la mise en place du projet du Boisé. Ainsi, afin de réduire ces dérives, les participants jugent qu'il serait bénéfique de cesser de donner des contraventions reliées à la consommation de stupéfiant ou d'alcool 
dans le Boisé. Ils proposent également que la Ville de Gatineau révise certains règlements municipaux ou, du moins, qu'elle soit plus flexible quant à leur application auprès des populations devant utiliser l'espace public comme milieu de vie. Le profilage social étant, à leur avis, une problématique systémique, les solutions doivent être pensées et mises en œuvre à un niveau macrosocial afin d'avoir des répercussions concrètes sur le terrain.

\section{Bibliographie}

BROUSSELLE,Astrid, et collab. (2009). L'évaluation : concept et méthode, Québec, Presse de l'Université de Montréal, $336 \mathrm{p}$.

CAMPEAU, Paule (2000). «La place des facteurs structurels dans la production de l'itinérance », dans Danielle Laberge (dir.), L'errance urbaine, Sainte-Foy, Éditions MultiMondes, p. 49-69.

CONTANDRIOPOULOS, André-Pierre, et collab. (2000). "L'évaluation dans le domaine de la santé : concepts et méthodes ", Revue d'épidémiologie et de santé publique, № 48, p. 517-537.

CYR, Kim (2011). Trajectoires et expériences itinérantes dans la région de l'Outaouais, [thèse de maîtrise], Ottawa, Université d'Ottawa, 118 p.

LE BOSSÉ, Yann (2003). "De "l'habilitation" au "pouvoir d'agir" : vers une appréhension plus circonscrite de la notion d'empowerment ", Nouvelles pratiques sociales, Vol. 16, № 2, p. 30-51.

ROY, Shirley (1995). "L'itinérance : forme exemplaire d'exclusion sociale? ", Lien social et Politiques, $\mathrm{N}^{\circ} 34$, p. $73-80$.

VILLE DE GATINEAU (2006). «Communauté 70 du Vieux-Hull Ouest, Portrait des communautés de l'Outaouais ", réf. du $1^{\text {er }}$ avril 2015, http://www.latino.qc.ca/Communautes/pdf/fascicule/ Fascicule_70.pdf 